INPLASY PROTOCOL

To cite: Ponvilawan et al. Efficacy of Targeted Immunotherapy as Induction or Salvage Therapy in Acute Lymphoblastic Leukemia: A Systematic Review and MetaAnalysis. Inplasy protocol 202170011. doi: 10.37766/inplasy2021.7.0011

Received: 04 July 2021

Published: 04 July 2021

Corresponding author: Weerapat Owattanapanich weerapato36733@gmail.com

Author Affiliation:

Division of Hematology, Department of Medicine, Faculty of Medicine Siriraj Hospital, Mahidol University.

Support: None.

Review Stage at time of this submission: Completed but not published.

Conflicts of interest:

None declared.

\section{Efficacy of Targeted Immunotherapy as Induction or Salvage Therapy in Acute Lymphoblastic Leukemia: A Systematic Review and Meta-Analysis}

\author{
Ponvilawan, B1; Vittayawacharin, P2; Tunsing, P3; \\ Owattanapanich, W4.
}

Review question / Objective: To summarize the available data and compare the efficacies and toxicities of cluster of differentiation(CD)-targeted immunotherapy and conventional chemotherapy during the induction or salvage therapy in patients with acute lymphoblastic leukemia.

Condition being studied: Acute lymphoblastic leukemia.

Eligibility criteria: To qualify for the meta-analysis, studies had to be either randomized, controlled studies (RCTs) or cohort studies (prospective or retrospective), and have 2 groups of ALL patients: 1 group which received CD-targeted immunotherapy for the induction or salvage therapy, and another group which did not receive any CD-targeted immunotherapy for the induction or salvage therapy. For both groups, the studies needed to have reported our primary outcomes of interest-overall survival (OS), relapse-free survival (RFS), or complete remission (CR)-by reporting the number of patients in each group for each outcome.

INPLASY registration number: This protocol was registered with the International Platform of Registered Systematic Review and Meta-Analysis Protocols (INPLASY) on 04 July 2021 and was last updated on 04 July 2021 (registration number INPLASY202170011).

\section{INTRODUCTION}

Review question / Objective: To summarize the available data and compare the efficacies and toxicities of cluster of diff erentiation (CD) - targeted immunotherapy and conventional chemotherapy during the induction or salvage therapy in patients with acute lymphoblastic leukemia.

Rationale: Acute lymphoblastic leukemia (ALL) is a malignant hematologic neoplasm of the lymphoid progenitor cells. It is the most common form of leukemia in children 
and the second most common form of acute leukemia in adults. However, the 5year overall survival rate of adult ALL patients is low $(35 \%-45 \%)$. As a wellestablished cornerstone of the treatment of ALL, several conventional chemotherapeutic regimens generally have fair outcomes. However, they had poorer outcomes for high-risk genetic profile cases, such as Philadelphia chromosome (Ph)-like ALL with 5-year event-free survival of $20 \%$ and 5 -year overall survival of $23 \%$. Immunotherapy has recently contributed to major advances in the treatment of various hematologic malignancies as a result of several surface antigens being expressed on malignant cells, including ALL. Multiple agents which target cell surface proteins, especially cluster of differentiation (CD) proteins such as CD19, CD20, and CD22, have been proved to generate favorable outcomes in ALL patients. However, there has been no systematic review and metaanalysis comparing the efficacies and toxicities of chemotherapy alone with those of immunotherapy with or without conventional chemotherapy.

Condition being studied: Acute lymphoblastic leukemia.

\section{METHODS}

Search strategy: Two investigators individually searched all published studies indexed in the EMBASE, MEDLINE, PubMed, and ScienceDirect databases from inception to June 2021. Search terms are as follow: EMBASE Database: 1. 'acute lymphoblastic leukemia'/exp OR 'acute lymphoblastic leukemia' 2. 'rituximab'/exp OR 'rituximab' 3. 'obinutuzumab'/exp OR 'obinutuzumab' 4. 'ofatumumab'/exp OR 'ofatumumab' 5. 'cd20 antibody'/exp OR 'cd20 antibody' 6. 'blinatumomab'/exp OR 'blinatumomab' 7. 'cd19 antibody'/exp OR 'cd19 antibody' 8. 'epratuzumab'/exp OR 'epratuzumab' 9. 'inotuzumab ozogamicin'/ exp OR 'inotuzumab ozogamicin' 10. /cd22 antibody'/exp OR 'cd22 antibody' 11. 'alemtuzumab'/exp OR 'alemtuzumab' 12. 'cd52 antibody'/exp RO ‘cd52 antibody' 13. 'combotox'/exp OR 'combotox' 14. 'moxetumomab pasudotox'/exp OR 'moxetumomab pasudotox' 15. 'denintuzumab mafodotin'/exp OR 'denintuzumab mafodotin' 16. 'coltuximab ravtansine'/exp OR 'coltuximab ravtansine' 17. 'immunochemotherapy'/exp OR 'i m munochemotherapy' 18 . 'immunotherapy'/exp OR 'immunotherapy' 19. \#2 OR \#3 OR \#4 OR \#5 OR \#6 OR \#7 OR \#8 OR \#9 OR \#10 OR \#11 OR \#12 OR \#13 OR \#14 OR \#15 OR \#16 OR \#17 OR \#18 20. \#1 AND \#19; Ovid MEDLINE Database: 1. acute lymphoblastic leukemia.mp. or exp Precursor Cell Lymphoblastic LeukemiaLymphoma/ 2. acute lymphoid leukemia.mp. 3. rituximab.mp. or exp Rituximab/ 4. obinutuzumab.mp. 5. ofatumumab.mp. 6. cd20 antibody.mp. 7. blinatumomab.mp. 8. cd19 antibody.mp. 9. epratuzumab.mp. 10. inotuzumab ozogamicin.mp. 11. cd22 antibody.mp. 12. alemtuzumab.mp. or exp Alemtuzumab/ 13. cd52 antibody.mp. 14. combotox.mp. 15. moxetumomab pasudotox.mp. 16 . denintuzumab mafodotin.mp. 17. coltuximab ravtansine.mp. 18 . immunochemotherapy.mp. or exp Antineoplastic Combined Chemotherapy Protocols/ 19. immunotherapy.mp. or exp Immunotherapy/ 20. 1 or 221.3 or 4 or 5 or 6 or 7 or 8 or 9 or 10 or 11 or 12 or 13 or 14 or 15 or 16 or 17 or 18 or 1922.20 and 21; PubMed and ScienceDirect Databases: 1. "acute lymphoblastic leukemia" 2. "rituximab" 3. "obinutuzumab" 4. "ofatumumab" 5 . "blinatumomab" 6 . "epratuzumab " 7. "inotuzumab ozogamicin" 8. "immunochemotherapy" 9. "immunotherapy" 10. 2 OR 3 OR 4 OR 5 OR 6 OR 7 OR 8 OR 9 11. 1 AND 10.

Participant or population: Patients with acute lymphoblastic leukemia.

Intervention: CD-targeted immunotherapy with or without conventional chemotherapy in induction or salvage treatment.

Comparator: Patients with acute lymphoblastic leukemia who received conventional chemotherapy without CDtargeted immunotherapy in induction or salvage treatment. 
Study designs to be included: Randomizedcontrolled studies or cohort studies.

Eligibility criteria: To qualify for the metaanalysis, studies had to be either randomized, controlled studies (RCTs) or cohort studies (prospective or retrospective), and have 2 groups of ALL patients: 1 group which received CDtargeted immunotherapy for the induction or salvage therapy, and another group which did not receive any CD-targeted immunotherapy for the induction or salvage therapy. For both groups, the studies needed to have reported our primary outcomes of interest-overall survival (OS), relapse-free survival (RFS), or complete remission (CR)-by reporting the number of patients in each group for each outcome.

Information sources: All published studies indexed in the EMBASE, MEDLINE, PubMed, and ScienceDirect databases from inception to June 2021.

Main outcome(s): Overall survival (OS), relapse-free survival (RFS), or complete remission (CR). OS rate was defined as the percentage of patients who were still alive after diagnosis at a particular time of interest. RFS rate was defined as the proportion of patients who had CR without relapse or death at a particular time of interest. For the OS and RFS rates, the time of interest was the longest of 6 months, 1 year, 2 years, 3 years, or 4 years with available results in each study. CR was defined as a patient who had $<5 \%$ of bone marrow lymphoblasts, the absence of circulating blasts or extramedullary disease, an absolute neutrophil count $>1.0$ $\times 10^{\wedge} 9 / \mathrm{L}$, and a platelet count $>100 \times 10^{\wedge} 9 /$ L.

Additional outcome(s): Minimal residual disease (MRD) negativity and grade 3-5 febrile neutropenia. MRD negativity rate was defined as either $<0.01 \%$ bone marrow lymphoblasts, confirmed by cytometry or immunoglobulin, or T-cell receptor gene rearrangements in bone marrow samples. Febrile neutropenia was defined as an absolute neutrophil count <
$1.0 \times 10^{\wedge} 9 / \mathrm{L}$, and either a single temperature $>38.3^{\circ} \mathrm{C}$ or a sustained temperature of at least $38.0^{\circ} \mathrm{C}$ for more than 1 hour, according to the National Cancer Institute's Common Terminology Criteria for Adverse Events (version 4.0).

Data management: A standardized data collection form was used to extract the following data: last name of the first author; year of publication; number of participants in each group; number of male and female participants in each group; average participant age for each group; disease statuses of the included participants; chemotherapeutic regimens employed; type and dose of immunotherapy used during the induction; countries where the studies were conducted; study period (years); and number of participants with outcomes of interest in each group.

Quality assessment / Risk of bias analysis: Two investigators evaluated the quality of each study, using the Jadad scale for RCTs and the Newcastle-Ottawa scale for cohort studies.

Strategy of data synthesis: Review Manager 5.3 software (The Cochrane Collaboration, United Kingdom) was used for all statistical analyses. The pooled odds ratio (OR) and the associated $95 \%$ confidence interval $(\mathrm{Cl})$ was calculated by combining the effect estimates and $95 \% \mathrm{CI}$ from each study using the Mantel-Haenszel method. As it is likely that there could be interstudy heterogeneity, a random-effect model was used instead of a fixed-effect model. Statistical heterogeneity was determined using Cochran's $Q$ test, accompanied by the heterogeneity $\left(I^{\wedge} 2\right)$ statistic. The $\mathbf{I} \wedge 2^{2}$ statistical value quantifies the proportion of the total variation across studies which is explained by study heterogeneity more than by random chance, with 12 values of $0 \%-25 \%$ representing insignificant heterogeneity, $26 \%-50 \%$ low heterogeneity, $51 \%-75 \%$ moderate heterogeneity, and $>75 \%$ high heterogeneity. The presence of publication bias was to be visualized by a funnel plot if there was a sufficient number of eligible studies for the meta-analysis. 
Subgroup analysis: A subgroup analysis based on the target of immunotherapy, disease status, and age groups were also to be performed if there were enough studies.

Sensitivity analysis: None.

Language: No restriction.

Country(ies) involved: Thailand.

Keywords: Immunotherapy; Monoclonal antibody; Acute lymphoblastic leukemia; ALL; Meta-analysis.

Dissemination plans: Publication in a peerreviewed journal.

Contributions of each author:

Author 1 - Ben Ponvilawan - B.P. designed the study, collected the data, drafted the manuscript, prepared the final version, and read and approved the final manuscript.

Email: ben.ponv@gmail.com

Author 2 - Pongthep Vittayawacharin - P.V. designed the study, collected the data, made critical revisions, and read and approved the final manuscript.

Email: big667118@gmail.com

Author 3 - Pattaraporn Tunsing - P.T. designed the study and read and approved the final manuscript.

Email: sakorn.tun@mahidol.ac.th

Author 4 - Weerapat Owattanapanich W.O. designed the study, collected the data, performed the statistical analyses, made critical revisions, and read and approved the final manuscript.

Email: weerapato36733@gmail.com 